\title{
Cognitive Real-Time QoE Management in Video Streaming Services
}

\author{
Maria Torres Vega \\ IDLab-imec \\ Ghent University \\ Ghent, Belgium \\ Email: maria.torresvega@ugent.be
}

\author{
Antonio Liotta \\ University of Derby \\ Derby, UK \\ Email: A.Liotta@derby.ac.uk
}

\begin{abstract}
Satisfying the needs of users of online video streaming services requires not only to manage the network Quality of Service (QoS), but also to address the user's Quality of Experience (QoE) expectations. While QoS factors reflect the status of individual networks, they do not comprehensively capture the end-to-end features affecting the quality delivered to the user. In this situation, QoE management is the better option. However, traditionally used QoE management models require human interaction and have stringent requirements in terms of time and complexity. Thus, they fail to achieve successful performance in terms of real-timeliness, accuracy, scalability and adaptability. This dissertation work investigates new methods to bring QoE management to the level required by the real-time management of video services. In this paper, we highlight our main contributions. First, with the aim to perform a combined network-service assessment, we designed an experimental methodology able to map network QoS onto service QoE. Our methodology is meant to provide service and network providers with the means to pinpoint the working boundaries of their video-sets and to predict the effect of network policies on perception. Second, we developed a generic machine learning framework that allows deriving accurate predictive No Reference (NR) assessment metrics, based on simplistic NR QoE methods, that are functionally and computationally viable for real-time QoE evaluation. The tools, methods and conclusions derived from this dissertation conform a solid contribution to QoE management of video streaming services, opening new venues for further research.
\end{abstract}

Index Terms-Video Streaming Services, Quality of Experience, Machine Learning

\section{INTRODUCTION}

Satisfying users, devices and services' requirements is fundamental to successfully manage an ever-growing worldwide wireless network [1]. Particularly crucial is the case of video streaming services. New streaming protocols increase bandwidth requirements and transmission complexity., which are critical elements for service and network providers. Thus, being able to monitor such applications, and act upon them when improvements are needed, is an essential requisite. This task has traditionally been studied in the context of network Quality-of-Service (QoS) management. However, due to the variability in channel conditions, streaming over wireless networks incurs quality degradation, even when there is sufficient nominal capacity. This is because QoS factors reflect the status of individual networks but do not comprehensively capture the end-to-end features that affect the overall quality delivered to the user. To address these elements, Quality of Experience (QoE) management represents a much better proposition [1].

The overall goal of QoE management is to optimize the end-user QoE (end-user perspective), while making efficient use of network resources (current and future) and maintaining a satisfied customer base (provider perspective) [2]. Thus, successfully managing QoE for a specific application requires identifying factors affecting it (subjective and objective), from the point of view of various actors in the service provisioning chain, and understanding how these impact QoE. Resulting QoE models define the parameters to be monitored and measured, with the ultimate goal being effective QoE optimization strategies. The overall process of QoE management may be broken down into three general steps: (1) QoE modeling, (2) QoE monitoring and measurements, and (3) QoE optimization and control [2]. QoE management of real-time streaming services requires these steps to be performed in real-time, accurately and in an adaptable and scalable manner. In this situation, the traditionally used QoE management models and monitoring tools (subjected to human interaction) fail to achieve successful performance, due to their time and complexity requirements. The purpose of this dissertation has been to investigate new methods and algorithms capable of bringing QoE management to the level required by the realtime management of video streaming services.

In this paper we summarize the main contributions of this dissertation to the QoE management of video streaming services.

\section{OVERVIEW OF RESEARCH CONTRIBUTIONS AND PUBLICATIONS}

Our research contributions can be mapped on the QoE management loop (Figure 1). The purpose of this work has been to give answer to three important questions within the QoE management loop:

1) What is the effect of networks on the users perception of video streaming services (QoE Monitoring)?

Resilience of Video Streaming Services to Network Impairments: We proposed a general systematic experimental methodology to understand the influence of QoS conditions onto the perception of video streaming 


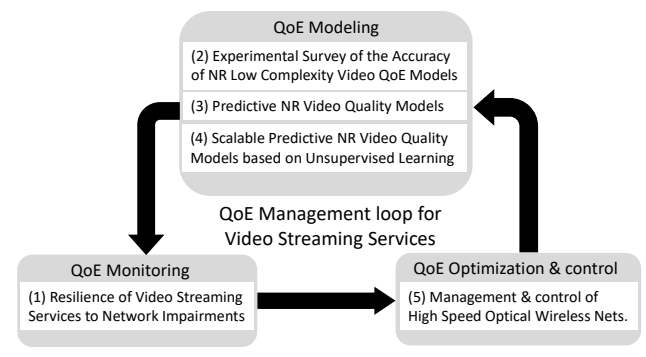

Fig. 1: QoE management loop with the contributions of this $\mathrm{PhD}$ thesis, located within the assigned functionality block in the QoE management loop.

services in a broad, general and systematic manner. Such an analysis is meant to provide video and network managers with the means to perform actions, both on the videos streams and the network control plane.

2) Can we measure the video streaming services degradation in real-time (QoE Modeling)?

The answer to this question is three folded:

a) Accuracy of QoE models for streamed video services: Our first step was to design and develop a methodology to analyze and characterize network impaired video-sets by means of the ground-truth quality (objective or subjective index attached) and the correspondent accuracy of simple No Reference (NR) video QoE metrics. This characterization provided new insights into the effects of network conditions onto the different aspects of streamed videos.

b) Cognitive QoE approaches: By means of cognitive supervised learning techniques, we demonstrated the possibilities behind combining simple (yet inaccurate) QoE metrics to obtain accurate (yet simple) ones.

c) Tackling the scalability of video QoE models. We explored the unsupervised deep learning world to devise a new method capable of providing realtime assessment based on no-reference characterization of the original non-impaired videos.

3) Is it possible to control the network to counter these effects (QoE control)?

QoE control is network dependent, i.e., the adjustments required to improve the perception are performed on the underlying network. This dissertation work has been financed by the European Research Council (ERC) Advanced Grant BROWSE [3], which proposes a novel wireless optical indoor communication system. This approach gave us the perfect network-example to apply network control optimizations. The control of the physical and MAC layer of this network were investigated and their integration with the QoE loop were discussed.

The following two Sections highlight the main responses to the first two challenges. Given the brief nature of this dissertation paper, the contributions to the control of the

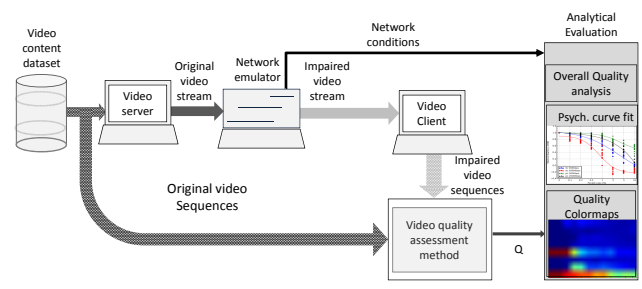

Fig. 2: General diagram for video quality assessment of networked videos.

network, will not be discussed in the remainder. Information regarding BROWSE and its management can be found in [1] [4], [5], [6].

\section{Publications}

A digital version of this dissertation is available online [3]. This research has led to 7 journal paper,s published in the IEEE Transactions on Broadcasting [7], [1], the IEEE Wireless Communication Letters [8], the IEEE Signal Processing Letters [9], the Springer journal Multimedia Tools and Applications [10], the Elsevier journal Signal Processing: Image communications [11] and the Emerald journal of Pervasive and Mobile Computing [12]. In addition, 12 papers have been included in the proceedings of international service management, and multimedia conferences [13] [14] [15] [16] [17] [18] [19] [4] [5] [6] [20] [21].

\section{WHAT IS THE EFFECT OF NETWORKS ON THE USERS PERCEPTION OF VIDEO STREAMING SERVICES?}

The driving motivation for this work was to provide a systematic analysis of the effect of QoS parameters on the end-user experience (QoE). Therefore, we engineered the system shown in Figure 2. To understand the direct effect of network impairments onto video services (i.e., without retransmissions), we picked the Real-time transport protocol (RTP) as transmission protocol. An RTP-video server streams to a RTP-video client, with a network emulator ${ }^{1}$. On video reception, the client performs an objective video quality assessment by means of a full-reference comparison between the original and the impaired data while the network emulator provides the real-time QoS. The QoS-QoS analysis consists of (1) overall quality degradation, (2) quality colormaps and (3) QoE-QoS psychometric curve fitting [7] per video class, where a video class is defined as the set of videos that suffer similar degradation due to networks. We chose RTP (Real-time transmission protocol) due to the fact that

Wireless networks suffer from instantaneous changes that translate into one out of the four basic QoS impairment categories (delay, jitter, packet loss and throughput constraints [22]). In this experimental evaluation, we assessed the isolated effect of each of these four impairments on the perceived quality of video streaming services.

QoE is inherently subjective [23]. However, subjective studies are unsuitable to perform real-time, large scale quality

\footnotetext{
${ }^{1}$ Hurricane II from PacketStorm, http://packetstorm.com/packetstormproducts/hurricane-ii-software/
} 


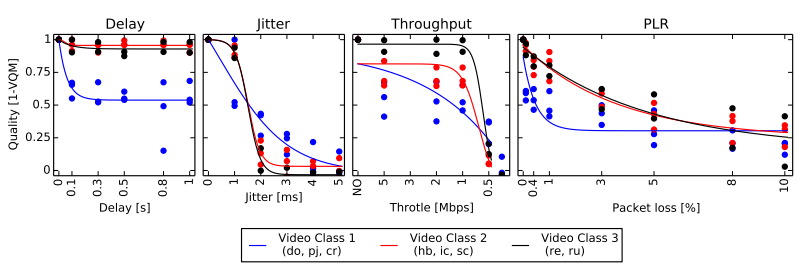

Fig. 3: Psychometric fits per class video type, compression and impairment for the RETRIEVE Video Quality Database.

analyses. On the other hand, objective metrics, which provide an assessment of the video degradation only based on the original and degraded material, are better suited. In our first investigation, we looked for an objective metric fitting the user's perception. Among all the possible full reference metrics we chose the Video Quality Model (VQM) [24] [25], [7].

In the next two subsections we highlight the main analyses performed using this methodology. First, we aimed to discover the most affecting QoS conditions. Then, Based on those results, we deepened the study of the most affecting conditions onto different video streaming services (Section III-B).

\section{A. Pinpointing the most affecting QoS conditions}

By means of the ReTRiEVED video quality database [22] (an MPEG2 video set which focuses on the effect of the four basic QoS impairments on Standard Definition videos), we aimed to pinpoint the most affecting QoS condition. This Section highlights the key discoveries. The full analysis can be found in [7].

Even if every video content type experiments degradation differently, out of our study, it was possible to spot similar behavioral patterns in terms of resilience against networks among the different videos. Three video clusters were identified, ranging from the videos that degrade the most, class 1 (blue in Figure 3 ), to videos suffering the least, class 3 (black in Figure 3), passing by an intermediate behavioral class, class 2 (red in Figure 3).

In general, while bandwidth throttle and packet loss provide a wide behavioral range, the temporal impairments (delay and jitter) affect the videos in the least. Delay only affects video class 1 , while the other classes show good resilience $(0.8$ quality with delays of 1 second) and the effect is flat (i.e., no significant difference between the lowest and the highest levels of delay). The jitter shows a generalized behavior for all the video classes: nearly full quality (or $50 \%$ for video class 1) up to $1 \mathrm{~ms}$ and full degradation for higher levels. As a general rule, in terms of bandwidth throttle, as the band is reduced to $1 \mathrm{Mbps}$, the quality is lost for most of the video classes. Regarding the packet loss, videos have reasonably good quality up to $3 \%$ drops. However, video class 1 starts loosing quality already at $0.4 \%$ (quality decreases down to roughly 0.6 ). Even if both impairments constraint the videos in a strong manner, the effect is heavily dependent on video type and condition. Thus, it is not easy to obtain general behavior

\footnotetext{
${ }^{2}$ Available online: http://www.comlab.uniroma3.it/retrieved.htm
}

rules with the assessment of only one video set. This made us think about deepening the study by creating and assessing our own bandwidth and packet loss impaired video sets.

\section{B. Resilience of $2 D$ and $3 D$ videos to Network Losses and Bandwidth Constraints}

In this second analysis, we made use of the experimental method presented in Figure 2 to generate our own bandwidth and packet losses impaired videosets. We generated the LIMP Video Quality Database (2D) and the 3D-HEVC-Net (3D) [7]

The LIMP Video Quality Dataset $[11]^{3}$ focuses on 2D H.264/MPEG4 videos. Ten original raw high quality videos obtained from the Live Quality Video Database [26] (10 seconds and 25fps) were compressed to H.264/MPEG4 with a resolution of $768 \times 432$ at 8 different bitrates $(64 \mathrm{kbps}, 640 \mathrm{kbps}$, $768 \mathrm{kbps}, 1024 \mathrm{kbps}, 2048 \mathrm{kbps}, 3072 \mathrm{kbps}, 4096 \mathrm{kbps}$ and 5120 kbps). The 80 original videos were streamed from server to client through the network of Figure 211 times, each iteration subjected to a different levels of packet loss, ranging from $0.5 \%$ to $5 \%$ in $0.5 \%$ steps and a final $10 \%$. The output is a set of 960 videos.

The 3D-HEVC-Net [7] studies the effects of packet loss and bandwidth on 3D stereoscopic videos. 10 original 3D Stereoscopic video sequences( 16 seconds, $1920 \times 1080,25 \mathrm{fps}$ ) [27] was compressed to H.265/HEVC ${ }^{4}$ at 6 compression levels (0.5Mbps, $1 \mathrm{Mbps}$, 1.5Mbps, 2Mbps, 3Mbps, and 4Mbps). The compressed sequences were merged into 6 streams (one per compression) and streamed through emulated network configured at 7 levels of packet loss $(0.1 \%, 0.2 \%, 0.5 \%, 1 \%$, $3 \%, 5 \%, 10 \%)$. Each streaming experiment was repeated 10 times for each bitrate and for each drop rate. On reception, the video sequences were split making a total of 4200 videos for analysis [21].

The videos of the two datasets were assessed in terms of their quality, and the performance was analyzed following Figure 2. Herein, we summarize the major highlights by means of the psychometric fits (Figure 4). The full analysis can again be found in [7].

From the point of view of the video types, even if the videos have different content types, it is again possible to find behavioral clusters, both for the 2D and the 3D lossy datasets. Four video clusters were identified for each of the datasets, ranging from very degraded to very resilient (one class per column in Figure 4 represents a video type either from the $2 \mathrm{D}$ or the $3 \mathrm{D}$ dataset). The videos belonging to each of the sets changes depending on the dataset. Additionally, given the broad range of bandwidth conditions, it was possible to spot four bandwidth compression classes, ranging from very low compression (4-5 Mbps) to very high compression (64Kbps for $2 \mathrm{D}$ and $0.5 \mathrm{Mbps}$ for $3 \mathrm{D}$ )

From the point of view of the compression, we found two general conclusions. First, as the bitrate decreases, the videos' robustness to network losses increases. This counter-intuitive

\footnotetext{
${ }^{3}$ Available online: https://www.tue.nl/index.php?id=53688

${ }^{4}$ http://x265.org/hevc-h265/
} 


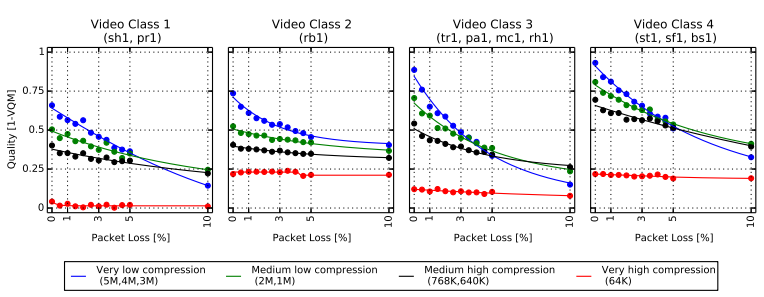

(a) Psychometric fits per class video types and compression of the LIMP Video Quality Database.

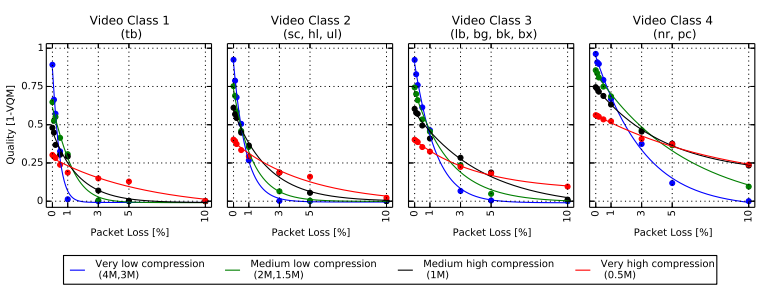

(b) Psychometric fits per class video types and compression of the Stereoscopic 3D videos of the 3D-HEVC-Net Video Quality Database.

Fig. 4: Psychometric fits for the videos of the two video datasets.

TABLE I: Video and compression class combinations depending on the network. The two selected sets 2D-SD (LIMP) and 3D-HD (3D-HEVC) are classified according to video and bandwidth classes. Packet loss are divided in five intervals.

\begin{tabular}{|c|c|c|c|c|c|c|}
\hline \multicolumn{2}{|c|}{$\begin{array}{l}\text { Videos } \\
\end{array}$} & \multicolumn{5}{|c|}{ Packet loss Interval [\%] } \\
\hline Set & Class & 0 & $0.1-1$ & $1-3$ & $3-5$ & $5-10$ \\
\hline \multirow{4}{*}{$\begin{array}{l}2 \mathrm{D} \\
\mathrm{SD}\end{array}$} & 1 & VLC & VLC & VLC & VLC & MLC \\
\hline & 2 & VLC & VLC & VLC & VLC & VLC \\
\hline & 3 & VLC & VLC & VLC & MLC & MHC \\
\hline & 4 & VLC & VLC & VLC & MLC & MHC \\
\hline \multirow{3}{*}{$\begin{array}{l}3 \mathrm{D} \\
\mathrm{HD}\end{array}$} & $\frac{1}{2}$ & $\begin{array}{l}\text { VLC } \\
\text { VL }\end{array}$ & $\begin{array}{l}\text { MLC } \\
\text { MHC }\end{array}$ & $\begin{array}{l}\text { VHC } \\
\text { VHC }\end{array}$ & $\begin{array}{l}\text { VHC } \\
\text { VHHC }\end{array}$ & $\begin{array}{l}\text { VHC } \\
\text { VHC }\end{array}$ \\
\hline & 3 & VLC & VLC & MHC & VHC & VHC \\
\hline & 4 & VLC & VLC & MLC & MHC & VHC \\
\hline
\end{tabular}

effect can be seen both for the 2D and 3D videos of Figure 4. Medium and lower bitrates suffer lower degradation than the higher variants as the losses in the network increase. In the case of 2D video, the crossing occurs between $3-5 \%$ while in the case of the $3 \mathrm{D}$ videos, the effect is more dramatic and in most of the video classes, higher bitrate variants show worse performance already for losses close to $1 \%$. Second, 3D videos are less resilient to packet loss. Unlike, 2D videos, where, in general, the quality gradually drops as the losses increase, 3D videos show extreme drops, going from qualities of 0.9-0.8 at $0.1 \%-0.2 \%$ to roughly 0.5 for $1 \%$ packet loss and down to 0.25 for $3 \%$. This is due to the higher resolutions of the videos and bitrates, apart from the fact that $3 \mathrm{D}$ videos transport more complex information within the two views.

To illustrate the power behind the presented analysis, let's imagine a video service provider, storing video content both on 2D-SD (LIMP) and 3D-HD (3D-HEVC-Net). To adapt the videos to the network and client requirements, the content and compression available could be classified. Based on the 16 classes of Figure 4, the provider could produce Table I. Tables such as this one will provide valuable to network and service providers, to pursue resource efficiency and user satisfaction (trades-offs). For example, if a client were to request a 3DHD video of class 2, this would only be transferred at the highest quality, if the network is clean ( $0 \%$ loss). Where any disturbances were sensed on the network, the video provider could decide to stream the video at a higher compression rate, to prevent loss of quality on the client side. Taking the network perspective, if the requested 3D-HD video class 2 were set to the maximum transmission rate at client's request, the network manager would be required to ensure for the network to remain clean (i.e., having virtually no losses) during the whole streaming session. If this were not guaranteed, other improving actions, such as prioritizing the client's traffic or increasing transmission power, would be needed.

\section{CAN WE MEASURE THE VIDEO STREAMING SERVICES DEGRADATION IN REAL-TIME?}

In a real-time QoE management system, a client, connected to a video server, should be able to provide online assessments of the received content. These, could, in turn, be used as feedback to the video and network services. In the best case, the client's method would require close to no information from the server. Furthermore, given the variety of clients (HD video systems, personal computers, smart-phones, etc.), the computational complexity of the client's method would be minimal.

As we saw in the previous Section, VQM provides an accurate measure of video degradation. Yet, VQM requires access to the original material and, tends to be very computationally heavy. Thus, it is unfit for real-time evaluation, and better suited as a benchmark. Reduced Reference (RR) and NR metrics, on the other hand, perform their assessment based only on the received material and the network conditions. Therefore, they are the most adequate solution, at least in terms of timeliness and efficiency [12], [28]. However, their accuracy in terms of correlation to subjective studies and full reference metric is still an open subject of discussion.

The purpose of this research track has been to design a general, scalable video QoE model capable of measuring video quality degradation accurately and in real-time. In the next subsections the main contributions of this track are highlighted. The LIMP Video Quality Database was used for these studies and the accuracy of the models was assessed by means of the Pearson Correlation Coefficient (PCC) to VQM along the level of packet loss.

\section{A. Accuracy of No-Reference Low Complexity Metrics}

Our first step in this direction was to evaluate the performance of well-known state-of-the-art real-time NR metrics. Therefore, we picked a representative set of eight simple NR real-time metrics ( 6 on the pixel and 2 on the bitstream level) [12].

On the one hand, The pixels of video frames can degrade in terms of sharpness (blur, average and ratio) and cleanness (noise, average and ratio) [17]. In addition, pixels have the tendency to develop blocks in which the image is not clear and cannot be processed, i.e., blockiness [29]. Moreover, due 


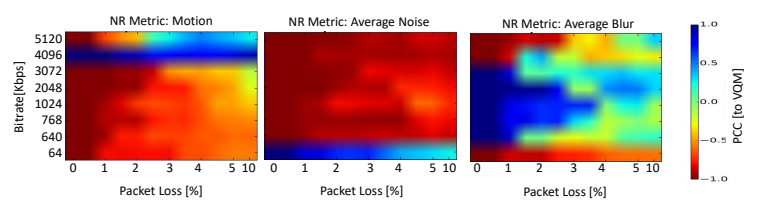

Fig. 5: PCC colormaps of three NR metrics (Noise Average, Motion and Average Blur) of the video pr1 of the LIMP Video Quality Database.

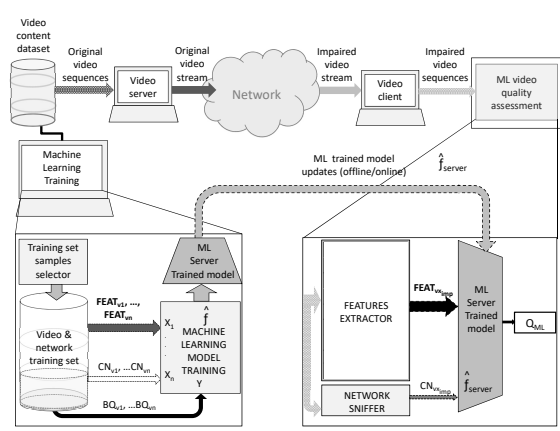

Fig. 6: General diagram for machine learning-based video quality assessment.

to limited bandwidth, the encoder reduces the frame rate, and other errors might lead to repetitions, drops or displays of extra frames. These impairments lead to a perceptual degradation called jerkiness [30]. On the other hand, bitstream parameters regarding the video scene composition have been demonstrated to affect quality. Among these, scene complexity and video motion have proven to correlate well with video quality [12].

We evaluated the LIMP database with these 8 NR metrics. To explore the working limits of the various measurements, we analyzed the different video types individually, with particular attention to compression level ( $\mathrm{Y}$ axes) and packet loss ( $\mathrm{X}$ axes). Figure 5 provides an example of three of these metrics for the video type 'pr1'. In it, maximum correlation to VQM is shown in dark blue and maximum anti-correlation in dark red. The full set of results is available in [12].

These results showed that there is no metric that could be used in a general manner. In the example (Figure 5), motion shows high levels of anti-correlation for most of the videos, except for the $4096 \mathrm{kbps}$ variant, while the average noise correlates only in the case of $64 \mathrm{Kbps}$. Blur, on the other hand, could be used up to certain values of packet loss (between 2-4\%) for 6 of the compression levels. However, the anti-correlation is extreme for very low and very high bitrates.

It was encouraging, though, that specific blue areas (well correlated) emerged for all the NR metrics under scrutiny. Armed with these results we moved on to the study and design of hybrid metrics that would combine the individual strengths.

\section{B. Cognitive No-Reference Real-Time Video Quality Models}

To develop an hybrid metric combining the strengths of individual simplistic NR metrics, we took the Machine Learning (ML) path. ML tools have demonstrated to provide the required accuracy enhancement of the quality assessment for

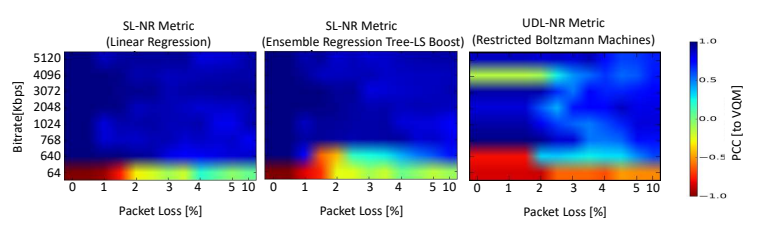

Fig. 7: PCC correlation diagrams for three machine learning based models: (a) Linear Regression, (b) Ensemble Regression Trees - LSBoost, (c) Restricted Boltzmann Machines.

real-time client based methods [31]. Our contribution consisted of a generic ML framework (Figure 6), that allows deriving a predictive NR assessment metric [11]. As with any predictionbased method, the accuracy of the model substantially depends on the characteristics of the training dataset. In our case, the training set is composed by a number of samples stored in the server. Each sample includes the eight NR features presented in the previous Section and two network parameters (packet loss and received bitrate). The output consists of the ground truth quality (VQM in this case). This training set is used to maintain the quality prediction function, which is employed on the client side to compute the predictive quality assessment metric. At service launch, the service provider will already have a representative video set; thus an initial model can be constructed (and made available to the client). When a completely new video type is added, the prediction model will be less accurate. Yet, over time, the model will be updated based on new types and, what is more important, the chances of getting new video types will rapidly diminish. In this way, the server runs a process in the background in which the ML model is trained with the available video samples, and new models $\left(\hat{f}_{\text {server }}\right)$ are uploaded to the clients (on a continuous or periodic basis). On the other end of the transmission link, the video client employs the ML model trained by the server, to generate its prediction-based quality metric $\left(Q_{M L}\right)$. During a streaming session, the client characterizes the incoming video in terms of the NR features and real-time network conditions, matching this information against the prediction model to generate the quality estimation. We performed two types of assessments with this framework:

\section{1) Supervised Learning-based methods}

We first evaluated our approach using 9 different Machine Learning methods, ranging from very simple supervised learning-based white boxes, such Multiple Linear Regression or Regression Tree, to more complex supervised learning-based black boxes such as Convolutional Neural Networks. As a dataset, we again used the LIMP video quality database. In general, we obtained an accuracy of close to $96 \%$ with Supervised Learning algorithms, in particular with the Ensemble Regression Trees (Least Square Boost variant). However, most of the metrics showed promising results. These results can be found in [11]. In order to compare the performance of these metrics with the ones presented in Section IV-A, Figure 7 shows an example of these 
metrics' performance (first and second colormaps, linear regression and ensemble regression tree, respectively). While in Figure 5 red (ant-correlation) was predominant, in Figure 7 blue reigns over the different colomaps with some isolated exceptions. These results demonstrated the strength of our cognitive approaches.

\section{2) Unsupervised Learning-based methods}

One known problem of supervised learning methods is the lack of scalability and adaptability. The fact that every new sample entering the training set needs to be benchmarked introduces both complexities and unwanted delays. Unsupervised Deep Learning (UDL) provides a promising alternative. We evaluated UDL by means of the well-known Restricted Boltzmann machines (RBM) [9]. Therefore, we used the system of Figure 6 to design an RBM-based NR method. Again we evaluated on the LIMP database. We obtained an accuracy close to $80 \%$ [9], [10] (last colormap of Figure 7), while training only on the 10 original videos (before compression). This is a remarkable result, taking into account that UDL does not require the groundtruth quality to train the systems, which allows for very scalable solutions.

\section{CONCLUSION}

QoE management has the potential to provide the best quality assessment in online video streaming services. However, the current QoE management models fail to achieve real-time, accurate, scalable and adaptable performance, due to their time and subjective interaction requirements. The purpose of this dissertation work has been to research tools, algorithms and metrics to bring QoE management up to speed to the requirements of real-time video streaming services.

\section{REFERENCES}

[1] M. Torres Vega, C. Perra, F. De Turck, and A. Liotta, "A Review of Predictive Quality of Experience Management in Video Streaming Services," IEEE Transactions on Broadcasting, vol. 64, no. 2, pp. 432445, June 2018.

[2] S. Barakovic and L. Skorin-Kapov, "Survey and Challenges of QoE Management Issues in Wireless Networks," Journal Comp. Netw. and Communic., vol. 2013, pp. 165 146:1-165 146:28, 2013.

[3] M. Torres Vega, Cognitive Management and Control of High Speed Indoor Optical Wireless Networks, 2017. [Online]. Available: https://research.tue.nl/en/publications/ cognitive-management-and-control-of-high-speed-indoor-optical-wir

[4] M. Torres Vega, J. Famaey, A. Koonen, and A. Liotta, "Resource Allocation in Optical Beam-steered Indoor Networks," in IEEE NOMs, Istanbul, Turkey, April 2016

[5] A. Koonen, C. Oh, A. Khalid, K. Mekonnen, M. Torres Vega, and E. Tangdiongga, "2D beam-steered high-capacity optical wireless communication," in Photonics SUM, Newport Beach, CA, USA, July 2016.

[6] A. Khalid, M. Torres Vega, K. Mekonnen, Z. Cao, A. Liotta, and A. Koonen, "Real Time 10Gb-Ethernet Transmission over 2D Indoor Passive Beam Steered Optical Wireless System based on High Port Arrayed Waveguide Gratings," in 42nd ECOC, Germany, Sept. 2016

[7] M. Torres Vega, C. Perra, and C. Liotta, "Resilience of Video Streaming Services to Network Impairments," IEEE Transactions on Broadcasting, vol. 64, no. 2, pp. 220-234, June 2018.

[8] M. Torres Vega, A. M. J. Koonen, A. Liotta, and J. Famaey, "Fast Millimeter Wave Assisted Beam-Steering for Passive Indoor Optical Wireless Networks," IEEE W. Comm. Let., vol. PP, no. 99, 2017.
[9] M. Torres Vega, D. C. Mocanu, J. Famaey, S. Stavrou, and A. Liotta, "Deep learning for quality assessment in live video streaming," IEEE Signal Processing Letters, vol. 24, no. 6, pp. 736-740, 2017.

[10] M. Torres Vega, D. C. Mocanu, and A. Liotta, "Unsupervised deep learning for real-time assessment of video streaming services," Multimedia Tools and Applications, pp. 1-25, 2017.

[11] M. Torres Vega, D. C. Mocanu, S. Stavrou, and A. Liotta, "Predictive No-Reference Assessment of Video Quality," Signal Processing: Image Communication, vol. 52, pp. 20-32, march 2017.

[12] M. Torres Vega, V. Sguazzo, D. C. Mocanu, and A. Liotta, "An experimental survey of no-reference video quality assessment methods," Int. J. Perv. Comp. and Comm., vol. 12, no. 1, pp. 66-86, 2016

[13] D. Mocanu, A. Liotta, A. Ricci, M. Vega, and G. Exarchakos, "When does lower bitrate give higher quality in modern video services?" in IEEE NOMS, Krakow, Poland, May 2014, pp. 1-5.

[14] M. Torres Vega, S. Zou, E. Tangdiongga, A. Koonen, and A. Liotta, "HD-video streaming over an inexpensive in-building radio-over-MMF system," in Proceedings of the 19th Annual Symposium of the IEEE Photonics Society, Entschede, The Netherlands, nov. 2014.

[15] M. Torres Vega, S. Zou, D. Mocanu, E. Tangdiongga, A. Koonen, and A. Liotta, "End-to-End Performance Evaluation in High-Speed Wireless Networks," in CNSM, Rio de Janeiro, Brazil, nov. 2014.

[16] M. Torres Vega, D. C. Mocanu, R. Barresi, G. Fortino, and A. Liotta, "Cognitive streaming on android devices," in in proc. of the 1st. IEEE/IFIP IM 2015 International Workshop on Cognitive Network \& Service Management, Ottawa, Canada, May 2015.

[17] M. Torres Vega, E. Giordano, D. C. Mocanu, and A. Liotta, "Cognitive no-reference video quality assessment for mobile streaming services," in QoMex, May 2015.

[18] D. C. Mocanu, M. Torres Vega, and A. Liotta, "Redundancy reduction in wireless sensor networks via centrality metrics," in Proceedings 15th IEEE International Conference on Data Mining Workshop (ICDMW), Atlantic City, New Jersey, november 2015.

[19] M. Torres Vega, V. Sguazzo, D. C. Mocanu, and A. Liotta, "Accuracy of no-reference quality metrics in network-impaired video streams," in MoMM, Brussels, Belgium, Dec. 2015.

[20] M. Torres Vega, D. Constantin Mocanu, and A. Liotta, "A Regression Method for real-time video quality evaluation," in MoMM 2016, Singapore, Singapore, November 2016.

[21] M. Torres Vega, D. C. Mocanu, C. Perra, and A. Liotta, "Effects of lossy networks on stereoscopic 3d-video streams," in IEEE BMSB, Cagliari, Italy, June 2017.

[22] P. Paudyal, F. Battisti, and M. Carli, "Impact of video content and transmission impairments on quality of experience," Multimedia Tools and Applications, vol. 2016.

[23] P. Le Callet, S. Moeller, A. Perkis, and eds., "Qualinet white paper on definitions of Quality of Experience," in COST Action IC 1003, Laussanne, Switzerland, 2012.

[24] M. H. Pinson and S. Wolf, "A new standardized method for objectively measuring video quality," IEEE TBC, vol. 50, no. 3, pp. 312-322, Sep. 2004.

[25] S. Chikkerur, V. Sundaram, M. Reisslein, and L. J. Karam, "Objective Video Quality Assessment Methods: A Classification, Review, and Performance Comparison," IEEE TBC, vol. 57, no. 2, pp. 165-182, 2011.

[26] K. Seshadrinathan, R. Soundararajan, A. C. Bovik, and L. K. Cormack, "Study of subjective and objective quality assessment of video," Trans. Img. Proc., vol. 19, no. 6, pp. 1427-1441, Jun. 2010.

[27] M. Urvoy, M. Barkowsky, R. Cousseau, Y. Koudota, V. Ricorde, P. Le Callet, J. Gutierrez, and N. Garcia, "NAMA3DS1-COSPAD1: Subjective video quality assessment database on coding conditions introducing freely available high quality 3D stereoscopic sequences," in QoMEX, 2012, pp. 109-114.

[28] M. Shahid, A. Rossholm, B. Lövström, and H. Zepernick, "No-reference image and video quality assessment: a classification and review of recent approaches," EURASIP J. Im. and Vid. Proc., vol. 2014, p. 40, 2014.

[29] C. Perra, "A low Computational Complexity Blockiness Estimation Based on Spatial Analysis," in IEEE 22nd Telecomm. Forum, 2014.

[30] S. Borer, "A model of jerkiness for temporal impairments in video transmission," in QoMEX, 2010.

[31] N. Staelens, G. V. Wallendael, K. Crombecq, N. Vercammen, J. D. Cock, B. Vermeulen, R. V. de Walle, T. Dhaene, and P. Demeester, "No-Reference Bitstream-Based Visual Quality Impairment Detection for High Definition H.264/AVC Encoded Video Sequences," IEEE TBC, vol. 58, no. 2, june 2012. 